\title{
LA ORDENACIÓN DE LAS REGLAS Y LA FONOLOGÍA LÉXICA 1
}

\author{
PALOMA GARCÍA-BELLIDO \\ (U. A. M.)
}

\section{Introducción}

Desde la aparición de The Sound Pattern of English en 1968 se han venido desarrollando una serie de extensiones bastante importantes a la teoría de la fonología generativa allí esbozada. Todas estas extensiones tienen como proyecto en su investigación restringir la clase de gramáticas eliminando formulaciones ad hoc o hipótesis justificadas con criterios extrínsecos a la gramática ${ }^{2}$. Para ello unas investigaciones se han concentrado en articular con mayor precisión y coherencia una teoria de la estructura de la morfología y el léxico, como muestran, dentro del marco generativista, los trabajos de Halle (1973), Siegel (1974), Jackendoff (1975), Aronoff (1976), Allen (1978), Selkirk (1982) y otros.

Otras investigaciones han buscado restringir el conjunto de gramáticas poniendo restricciones a la aplicación de las reglas fonológicas y de ahí a las representaciones fonológicas, esto es a las representaciones subyacentes.

En esta dirección ha ido encaminada la sugerencia de Kiparsky $(1968=1973)$ de incluir en el componente fonológico La Condición de Alternancia (The Alternation Condition). La propuesta de incorporar ésta a la Condición de Aplicación Estricta de las reglas ciclicas, inicialmente formulada por Chomsky, Halle y Lukoff en 1956 y posteriormente tomada para aplicarla en la fonología por Brame $(1972,1974)$ y Kean (1974), se debe a Mascaró (1976). Éste, con la formulación de la Condición de Ciclo Estricto (The Strict Cycle Condition), consigue que, restringiendo la clase de reglas fonológicas cíclicas a un tipo particular de reglas, se deduzca la clase de gramáticas de una forma más apropiada ${ }^{3}$.

1 Este trabajo fue leído en el XIII Simposio de la Sociedad Española de Lingüística celebrado en Barcelona en diciembre de 1983 con el título de «La ordenación de las reglas y el ciclo fonológico».

2 Véase, a este respecto, Halle (1978).

3 Véase la discusión que desarrolla Kiparsky (1982, MS) para justificar la posible eliminación de la Condición de Ciclo Estricto. 
En esta misma dirección de restringir la aplicación de las reglas fonológicas está la Condición de Resto (The Elsewhere Condition) enunciada originalmente por Kiparsky (1973a) ${ }^{4}$ con la que se bloquea la aplicación de ciertas reglas generales en competencia con otras reglas particulares dadas determinadas condiciones previas.

En otro orden de cosas, la fonología métrica iniciada con la teoria métrica del acento en Lieberman y Prince (1977) y extendida en particular al estudio de la sílaba (Kiparsky, 1979; McCarthy, 1979; entre otros) y de la fonología no lineal o multiestratificada en general (Goldsmith 1976, McCarthy 1981, entre otros) ${ }^{5}$ ha contribuido también a enriquecer el conocimiento de la clase de operaciones fonológicas en las lenguas naturales.

Más recientemente Kiparsky (1982a, 1982b) ha desarrollado una teoria de fonología y morfología léxica en donde se interrelacionan en un léxico estructurado por niveles las principales contribuciones enunciadas más arriba de morfología de niveles, condiciones de aplicabilidad de reglas y fonología no lineal.

El propósito de este trabajo es demostrar que con la teoría léxica de Kiparsky, que enunciaremos con más detalle a lo largo de esta exposición, se puede reducir el conjunto de gramáticas del español. En concreto, sugerimos que una gramática del español no parece necesitar la inversión de orden de dos reglas - tal y como se propone en Harris (1973) - si analizamos ciertas regularidades e irregularidades verbales a la luz de la teoría léxica propuesta por Kiparsky.

Dividiremos la exposición en cuatro partes. En la primera expondremos el estado de la cuestión. En la segunda parte, una vez expuestos los principios y propiedades relevantes para nuestra discusión de la teoría léxica de Kiparsky, nos adentraremos a demostrar cómo ésta resuelve ciertas irregularidades y regularidades morfológicas nominales y verbales. Por último, comentaremos las implicaciones teóricas que ofrece nuestro análisis alternativo dentro de una teoría general del léxico.

\section{Estado de la cuestión: doble orden de aplicación de reglas}

J. W. Harris, en un trabajo escrito en 1970 y publicado en 1973, propone que en Español habría que estipular dos órdenes extrínsecas de aplicación para dos reglas fonológicas: la regla de Truncamiento formulada en (1) y la regla de Desvelarización enunciada en (2) ${ }^{6}$.

4 Kiparsky (1973a) atribuye una formulación explícita de la Condición de Resto a un texto del Mahābhasya (Kielhorn, vol. III, p. 69).

5 Véase el conjunto de artículos publicados en Van der Hulst \& Norval Smith (1982) como ejemplos de estas tendencias.

6 En realidad esta regla enunciada en (i) más abajo es, según Harris (1969), una simplificación de un conjunto de procesos que pueden representarse como en (ii)
(i) $k \rightarrow 5 /-\left[\begin{array}{c}\text {-cons } \\ - \text { post }\end{array}\right]$
(ii) $k \rightarrow t^{s} \rightarrow\left(d^{z} \rightarrow z \rightarrow\right) s$

En nuestra discusión utilizaremos (2) en vez de (i). Esto no altera para nada el hilo de nuestra argumentación. 
(1) $\mathrm{V} \rightarrow \varnothing /+\square+\mathrm{V}$

(2) $\mathrm{K} \rightarrow \theta-\left[\begin{array}{l}\text {-cons } \\ \text {-post }\end{array}\right]$

Esta doble ordenación da cuenta de ciertas alternancias consonánticas de la morfología del español. Sabemos que en el español aparecen alternancias entre $[k]$ y $[\theta]$. Por ejemplo en (3) la última consonante de la raíz se presenta como una oclusiva velar sorda en la palabra rotulada con la categoría $\mathrm{N}$, mientras que esta misma posición final de raíz aparece como fricativa interdental sorda en el verbo (3b).

(3a) [k o $k+\theta y o ́ n]_{N}$

(3b) $[\mathrm{K} \circ \theta+\dot{\mathrm{e}}+\operatorname{mos}]_{\mathrm{v}}$

Esta alternancia se ha explicado como derivada de la existencia de la regla (2), que se aplica a una raíz /KOK/ (Harris, 1969).

En (4a) la regla de Desvelarización no se puede aplicar al no encontrar la descripción estructural apropiada con lo que tenemos finalmente la misma realización oclusiva velar de la consonante subyacente. En (4b) Desvelarización se aplicaría al encontrarse el fonema subyacente $/ k$ / de la raíz seguido de una vocal anterior.

(4a) $[k \circ k+\theta y o n]_{N}$

Desv.

Otras

Reglas (4b) $[k \circ k+e+m o s]_{v}$

$\theta$

\section{$\theta$}

$$
[k \circ \theta \text { é } m \circ s]
$$

La existencia de las formas de Subjuntivo como las de (5a) y de la primera persona del singular del Presente de Indicativo como la de (5b), donde no hay una vocal anterior que siga a la consonante final de la raíz no constituyen un problema para la formulación de (2). La hipótesis es que hay una vocal temática presente en la representación subyacente.

(5a) Presente de Subjuntivo kwéசa

$k w e ́ \theta$ as

kwéøa

$k$ otámos

$k$ öáys

kwéசan (5b) Presente de Indicativo kwé $\theta$ o

Desvelarización tendría que aplicarse antes que la regla de Truncamiento puesto que si se hiciera después no se encontraría la descripción estructural apropiada para poderse aplicar: (6b), produciendo un resultado incorrecto. La posibilidad de interrelacionar con un orden extrínseco general estas dos reglas permite dar cuenta de las alternancias en (3) y (5) sin mayores complicaciones. 
(6a) $[k \circ k+e+o]_{v}$

Desv. $\quad \theta$

Trunc. $\varnothing$

O.R.

[k w é $\theta$ o] (6b) $[\mathrm{k}$ o $\mathrm{k}+\mathrm{e}+\mathrm{o}] \mathrm{v}$

Trunc. $\varnothing$

Desv.

O.R.

Sin embargo, el orden estipulado para dar cuenta de las alternancias similares a las de (3) y (5) se encuentra con problemas a la hora de tener que explicar otras alternancias como las de (7)

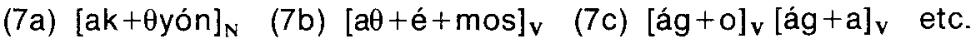

El problema que nos plantea la ordenación que aparece en (6a) es que predice un resultado incorrecto para la clase de verbos de (7), tal como se puede deducir de $(8)^{7}$.

(8a) [f a $\mathrm{k}+\mathrm{e}+\mathrm{o}]_{\mathrm{v}}$

Desv. $\theta$

Trunc. $\varnothing$

O.R.

*[á $\theta$ ol $]$ (8b) $[f \mathrm{a} k+e+o]_{v}$

Trunc. $\varnothing$

Desv.

O.R.

[á g o]

Sin embargo, el orden inverso de estas dos reglas, a saber: Truncamiento $>$ Desvelarización, nos da el resultado correcto para verbos como hacer y decir, como se puede deducir comparando las derivaciones (6) y (8).

Harris sugiere que «se podría especular» que las derivaciones excepcionales de hacer y decir «tiene (n) (P.G.B.) algún tipo de requisito léxico para que se invierta el orden normal de la pareja de reglas en cuestión" (Harris, 1975, p. 313).

En busca de una justificación para este requisito léxico de inversión de orden «normal» de dos reglas, se ha tenido en cuenta la tesis de Anderson (1969) por la que la aplicación máxima de dos reglas (cf. (6a)) favorecería el que hubiera un orden preferido «no marcado» frente a un orden «marcado» deducido de la no aplicación máxima de esas dos mismas reglas (Cf. (8b)). Esta tesis de Anderson queda fácilmente refutada para el español pues, como Harris observa, un verbo como marcar tiene un orden no marcado pero produce - contrariamente a lo esperado- una aplicación no máxima de las reglas en cuestión, como se ve en (9) en el Subjuntivo del verbo markar.

7 La forma /fak/ se encuentra en compuestos como satisfacer y comparte todas las formas del verbo hacer manteniendo la /f/ inicial. Aquí supondremos que para ambos casos la raíz es/fak/. Una regla tardía elidiria la / $t /$ inicial absoluta de palabra. Esto no influye para nada en nuestra argumentación. 
De hecho, hay razones para apoyar esta última hipótesis. Si se pudiera reducir la derivación morfológica de los deverbalizadores -ción a una sola operación en la que se insertará el sufijo en un contexto en el que el radical contiene una vocal temática (cf. (10)), entonces podríamos simplificar el conjunto de reglas morfológicas. El hecho de que la vocal temática sea cero o sea fonéticamente realizable vendría determinado por cada verbo, pero no por el sufijo deverbalizador en cuestión.

$$
\varnothing \rightarrow \text { yon } /\left[[\mathrm{X}]_{\sqrt{ }-}\right]_{\mathrm{N}}
$$

Donde $X$ contiene una vocal temática.

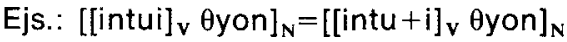

$$
\begin{aligned}
& {\left[[\text { sustitu }]_{\mathrm{V}} \text { } \theta \text { yon }\right]_{\mathrm{N}}=\left[[\text { sustitu }+\varnothing]_{\mathrm{V}} \text { } \theta \text { yon }\right]_{\mathrm{N}}=}
\end{aligned}
$$

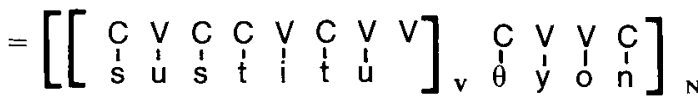

De esta forma no sería necesario estipular dos sufijos -ción y dos operaciones morfológicas, una de adición directa del sufijo a la raiz y otra de adición indirecta a la raíz, es decir a través de la vocal temática.

Lo mismo se podría decir de los sufijos de participio - $t$ que tienen formas temáticas como fre-i-d-o y formas atemáticas como fr-i-to, $m$ wé $r-t$-o. Nótese que la sonorización intervocálica del morfema de participio - $t$ aparece bloqueada, contrariamente a lo que esperaríamos, en frito/*frido. Es posible pensar que la derivación cero de la vocal temática en este caso es la responsable de la imposibilidad de aplicación de la regla de sonorización intervocálica ${ }^{9}$.

Si esto es plausible hay justificación independiente para pensar que las formas subyacentes de hacer y cocer en el Presente de Indicativo se añaden en la $1 .^{\text {a }}$ persona a una vocal temática de realización cero.

Esta alternativa no parece estar en contradicción con la teoría léxica enunciada por Kiparsky y que a continuación resumiremos muy brevemente en lo esencial para nuestra discusión.

9 Si la regla de sonorización intervocálica se enuncia en términos autosegmentales, esto es haciendo referencia a la estructura prosódica, el bloqueo de su aplicación puede ser fácilmente explicado. Supongamos que la regla en cuestión es como en (i):

(i)

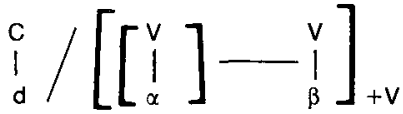

Donde $\alpha$ y $\beta$ son segmentos.

Entonces, esta regla puede aplicar en la estructura temática llena pero no en la vacía, pues en esta última la vocal autosegmental precedente no contiene un segmento.

(i)

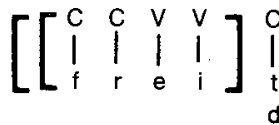

freido

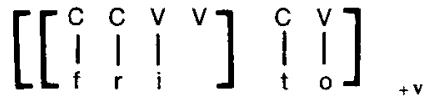

fríto

En un análisis segmental la regla de sonorización tendría que ser bloqueada en frito de alguna forma ad hoc. 


\subsection{La fonología y morfología léxica}

Como ya dijimos en la sección introductora la teoria de la fonología y la morfología léxica enunciada por Kiparky incorpora, entre otras cosas, una morfología de niveles ordenados. En (11) aparece esquemáticamente la estructura del léxico que organiza los procesos derivacionales e inflexionales de una lengua dada (Kiparsky, 1982b) (en Kiparsky, 1982a las flechas de nivel a nivel van de la fonología a la morfología).

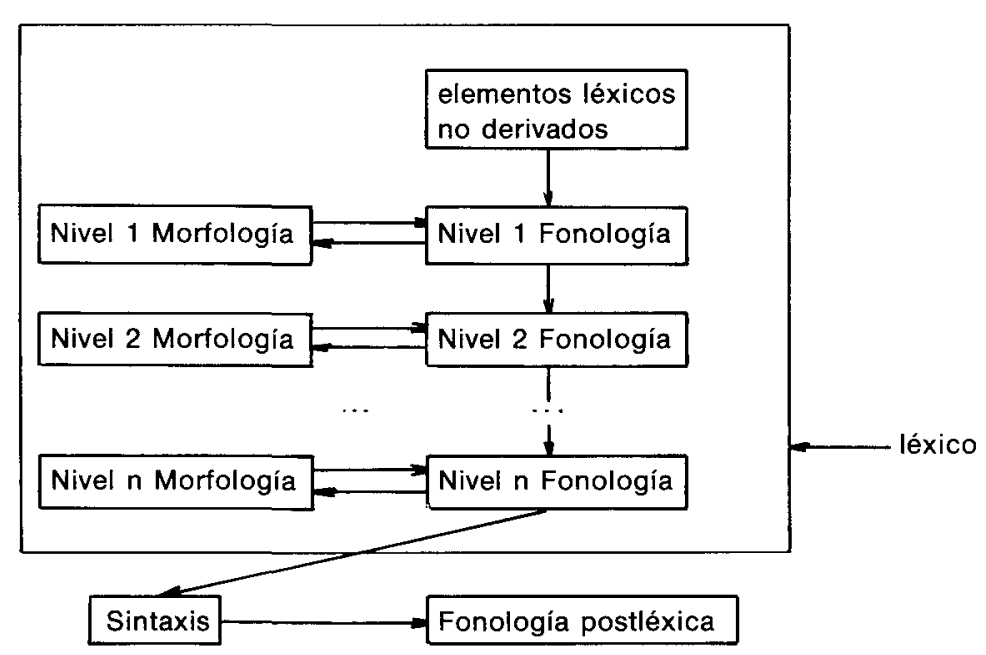

Las propiedades más pertinentes que podemos enunciar en este caso son las dadas en (12).

(12) Propiedades del léxico:

(i) Cada nivel está asociado a un conjunto de reglas fonológicas.

(ii) El orden en niveles define un orden posible de procesos morfológicos en la formación de palabras.

(iii) La salida de cada formación de palabra (cajas de la izquierda) se somete a las reglas fonológicas de su nivel.

(iv) La salida de las cajas de la derecha constituye el conjunto de elementos léxicos del lenguaje.

Este componente está sujeto a una serie de principios de los cuales sólo necesitaremos ahora enunciar los dados en (13).

(13)

(i) Los corchetes internos se borran al final de cada nivel.

(ii) La salida de cada capa de una derivación es una entrada léxica y tiene una regla de identidad que entra en un orden disyuntivo con otras leyes léxicas.

(iii) Las reglas léxicas están sujetas a la Condición de Resto (Elsewhere Condition).

Esta condición juega un papel crucial junto con el principio (13ii) para dar cuenta del efecto de «bloqueo». La definición de la Condición de Resto es enunciada por Kiparsky como sigue: 
(14) Condición de Resto (CR) (Elsewhere Condition; Kiparsky 1982b). Las reglas A,B en el mismo componente se aplican disyuntivamente a una forma $\varphi$ si y sólo si

(i) La descripción estructural de A (la regla especial) incluye propiamente la descripción estructural de $B$ (la regla general).

(ii) El resultado de aplicar A a $\varphi$ es distinto del resultado de aplicar B a $\varphi$. En ese caso, A se aplica primero y, si surte efecto, entonces $B$ no se aplica.

Una de las implicaciones más importantes de esta teoría reside en que La Condición de Ciclo Estricto puede quedar diluida en esta teoría léxica con la CR y el principio (13ii). Más adelante veremos con más detenimiento esta implicación.

Una vez expuesto el marco de trabajo al que nos vamos a ceñir pasaremos a justificar nuestro análisis alternativo para dar cuenta de las dos formas en discusión de verbos como cocer y hacer: cuezo / hago, cueza / haga, etc.

\subsection{Sobre algunos problemas de la regla de desvelarización}

Obsérvense las alternancias dadas en (15).

(15)

A

$\left[[\text { místik }]_{A}\right.$ o $]$

$\left[[\text { wertorík }]_{N} \mathrm{O}\right]$
B

$\left[\left[[m i s t i \theta]_{A} i s m\right]_{N}\right.$ o]

$\left[\left[[p w e r t o r i i \theta]_{N} e ́ n s\right]_{A} e\right]$
$\mathrm{C}$

$\left[\left[[m i s t i k]_{A} i s i m\right]_{A}\right.$ o $]$

$\left[\left[[\text { pwertorik }]_{N} e \tilde{n}\right]_{A}\right.$ O $]$

En Harris (1969) se propone que los casos como los de (15C) en los que el sufijo adjetivizador bloquea la regla de Desvelarización se consideren sufijos insertadores de una linde especial de palabra \#, mientras que los casos donde se produce desvelarización, como en (15B), la linde sea distinta a la de palabra, a saber de morfema +. Puesto que una convención estipula que la no mención de lindes en una regla implica la inclusión de la linde de morfema, la regla (2), que no menciona linde, se aplicaría tanto a formas no derivadas como a aquellas formas derivadas con la linde de morfema, pero no se aplicaría crucialmente a las formas derivadas con la linde de palabra ${ }^{10}$.

Aunque esta solución funciona, sin embargo adolece de poder explicativo ya que no puede evitar introducir elementos diacríticos como son los distintos tipos de lindes más arriba mencionados.

Puesto que la teoría léxica enunciada por Kiparsky nos permite organizar los procesos morfológicos y fonológicos por niveles, nada parece impedir el que los sufijos de linde de palabra sean organizados en un nivel distinto y posterior del de los sufijos de linde de morfema, consiguiendo con ello principalmente la supresión de diacríticos especiales para distinguir su comportamiento fonológico distinto. El comportamiento de estos sufijos y su interrelación con la regla de Desvelarización vendría pues explicada de una forma menos ad hoc si asumimos la siguiente organización léxica para el español.

10 Véase Harris (1969, 6.3.2.3.) para una argumentación relacionada con el ciclo, el acento y la diptongación. 
(16)

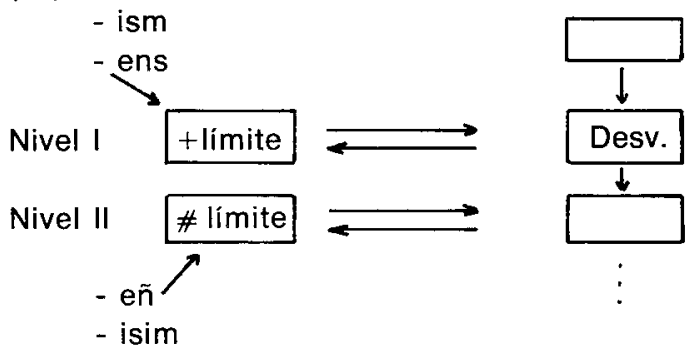

En (16) la regla de Desvelarización es una regla fonológica que se aplica sólo a las palabras que se forman en su nivel ${ }^{11}$, es decir a las que se han formado insertando, por ejemplo, -ismo, -énse por lo que no aparece en español (17a, 17b). Puesto que los sufijos -éño, -ísimo se insertan en el nivel Il y en ese nivel no hay desvelarización, no encontraremos formaciones posibles como (17c) ni como (17d). Esta organización predice que encontraremos formaciones posibles tanto como (17e) como (17f) y $(17 \mathrm{~g})$, y predice que no encontraremos $(17 \mathrm{~h})$.
a *pwertorikénse
b *mistikismo
c *mistïísimo
d *pwertorïéño

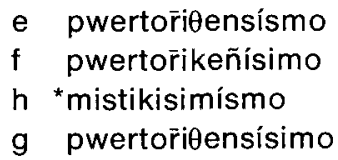

La teoría léxica nos permite, pues, dar cuenta de una serie de hechos del español sin necesidad de utilizar diacríticos.

Una vez justificado independientemente que hay dos niveles para la formación de palabras y que la Desvelarización no aplica a todos los niveles, pasaremos a articular nuestra alternativa con los casos verbales bajo discusión.

2.3. Reglas Especiales, Generales y la C.R.

Supongamos que el léxico tiene dos reglas morfológicas como las de (18a) y (18a)

$$
\varnothing \rightarrow \mathrm{V} /\left[\mathrm{V}_{\mathrm{n}}-\right]_{+} \mathrm{v} . \quad \text { Presente }\left\{\begin{array}{l}
\text { Subjuntivo } \\
\text { Indicativo, 1p.s. }
\end{array}\right\} \quad-\mathrm{i}
$$

Donde $V_{n}=$ hacer, decir.

11 Esta regla tiene el síndrome cíclico. No aplica a contextos no derivados pues encontramos excepciones: keso, kitar, etc. Por la CR predecimos que la Desvelarización no puede aplicar a estos contextos no derivados con lo que hay que dar a estos casos una representación subyacente $/ \mathrm{k}$. 
(18b)

$\varnothing \rightarrow\left\{\begin{array}{l}\mathrm{a}_{\alpha} \\ \mathrm{e}_{\beta} \\ \mathbf{i}_{\gamma}\end{array}\right\}^{\prime}\left\{\begin{array}{l}\mathrm{V}_{\alpha} \overline{{ }_{\beta}} \\ \gamma\end{array}\right\}+\mathrm{v}$

Donde $\alpha$ 1. ${ }^{\mathrm{a}}$ Conj., $\beta$ 2. ${ }^{\mathrm{a}}$ Conj., $\gamma$ 3. ${ }^{\mathrm{a}}$ Conj.

Las reglas de (18a) se aplicarían exclusivamente a los verbos hacer y decir en la 1p.s. del Presente de Indicativo y en todas las personas del Subjuntivo.

De acuerdo con la descripción estructural de (18a) estas reglas no podrían aplicarse al verbo cocer ni marcar, por ejemplo, por no estar contenidos en la clase de verbos $V_{n}$. Por otro lado, para dichos verbos especiales, tampoco ninguna otra persona del Presente de Indicativo podría ser analizada por la regla (18aii), así como tampoco ningún otro tiempo que no sea el Presente de Subjuntivo. Más crucialmente nada impide por el momento que (18b) se aplique a todos los tiempos y personas incluidos o no en (18a), de la misma forma que nada impide que (18b) se aplique a todos los verbos incluidos o no en (18a). Sin embargo, los verbos hacer y decir en su $1 .^{\text {a }}$ persona de Singular del Presente de Indicativo y en su tiempo de Presente de Subjuntivo no pueden ser analizados por la regla (18b) de acuerdo con la C.R. Veamos por qué.

Las descripciones estructurales de (18a), las reglas especiales incluyen propiamente a la de (18b), la regla general. Esto es, (18ai,ii) definen un subconjunto de los contextos de (18b). Por lo tanto, las reglas (18a) y (18b) cumplen el primer requisito de la C.R. Por otro lado, las salidas de (18a) y (18b) son distintas puesto que (18b) es diki y (18a) es dik. Con lo cual (18a) y (18b) cumplen el segundo requisito de la C.R.

Como el par de reglas de (18ai,ii) y (18b) cumplen ambos requisitos de la C.R., las reglas (18ai,ii) se aplicarán antes que la más general, y al surtir efecto bloquearán la aplicación de la regla (18b). Con lo que no tendremos ni *diki etc., ni formas con dos vocales temáticas siendo la primera de realización cero: *[[[dik]ø]i].

En este mismo ciclo la regla de Desvelarización encuentra su descripción estructural, por lo que se puede aplicar ${ }^{12}$. La consecuencia de la aplicación de las reglas morfológicas y fonológicas y de la C.R. puede verse en las derivaciones de (19) en las que contrastamos la aplicación de un caso específico, el Subjuntivo, y un caso general, el resto, excluyendo la $1 .^{a}$ persona del singular del Presente de Indicativo.

$\begin{array}{ccc}A & B & C \\ {[\text { dik }]_{V_{n}}} & {[k o k]_{\mathbf{v}}} & {[\text { dik }]_{V_{n}}} \\ {\left[[\text { dik }]_{\mathbf{V}_{\mathbf{n}}} \varnothing\right]_{\mathbf{P}, \mathbf{s},}} & - & -\end{array}$

12 Véase Mohanan \& Mohanan (1984) (p. 579) para una formulación explícita de esta misma hipótesis "In the absence of counterevidence, include the lowest possible stratum as the domain of the ruie (where «highest" = stratum 1)". 
C.R.

Salida $1^{\text {er }}$ Ciclo

O.R.

\section{$-\quad \theta$}

$[[\mathrm{dik}] \varnothing]_{\text {P.S. }}$

díga, etc. $[[k \circ \theta] e]_{v}$

kwé $\theta$ e, etc. $\left[\left[d_{i k}\right]_{v} \mathrm{i}\right]_{\mathrm{v}}$

$\theta$

$[[\operatorname{di\theta }] \mathrm{i}]_{\mathrm{v}}$

di $\theta$ e, etc.

En el siguiente ciclo morfológico es en el que se insertan los morfemas de Tiempo y Modo. Este proceso morfológico puede ejemplificarse con la formación de Presente de Subjuntivo en (20).

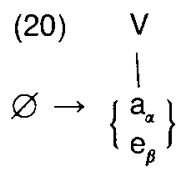

$$
\left[V_{\left\{\begin{array}{l}
\alpha \\
\beta
\end{array}\right\}}\right]+v \text {. Presente Subjuntivo }
$$

Donde $\beta=+1 .^{\mathrm{a}}$ Conj.

$$
\alpha=-1{ }^{a} \text { Conj. }
$$

La regla (20) inserta el morfema de Presente de Subjuntivo a todas las salidas del ciclo anterior. Sin embargo, a la salida de (19A) la Condición de Resto, que no parece haya que restringir a que se aplique sólo entre reglas de un mismo ciclo, hará que la aplicación de (18ai) bloquee la aplicación de (20) por haber surtido efecto la regla morfológica anterior. Por lo cual perdemos la posibilidad de insertar el morfema de Presente de Subjuntivo a estas formas, dejándonos con una forma incorrecta *dik para la $1{ }^{a}$ persona de singular del Presente de Subjuntivo, por poner un ejemplo.

Por lo tanto, no es suficiente tener sólo una regla de formación de Tiempo y Modo de Presente de Subjuntivo. Parece necesario, dentro de este modelo, enunciar una regla especial de formación que inserte los morfemas de P.S. para ciertos verbos específicos; en concreto, aquellos que tienen derivación cero de vocal temática. Esta regla se puede formular como en (21)

$$
\varnothing \rightarrow\left\{\begin{array}{l}
\mathrm{a}_{\alpha} \\
\left.\mathrm{e}_{\beta}\right\} \quad, \quad\left[\mathrm{V}_{\mathrm{n}}^{\alpha} \underset{\beta}{\alpha}\right\}
\end{array}\right]+\mathrm{v}, \text { P.S. }
$$

Donde $\mathrm{V}_{\mathrm{n}}=$ hacer, decir.

$$
\begin{aligned}
& \beta=+1 .^{a} \text { Conj. } \\
& \alpha=-1 .^{a} \text { Conj. }
\end{aligned}
$$

Según la C.R., las reglas (20) y (21) entrarán en una relación disyuntiva por la que la regla (21) se aplicará a las salidas de (19A): [[dik] $\varnothing$ ]. Puesto que (21) surte efecto la regla más general (20) no podrá aplicarse. Veamos por qué. La descripción estructural de (21) incluye propiamente la de (20): (21) define un subconjunto de los 
contextos de (20) cumpliendo pues, el primer requisito de la C.R. Las salidas de (21) y (20) son distintas. En la salida de (21) no aparece una vocal temática detrás de la raíz; [[[dik]]a], mientras que en la de (20) sí aparece una vocal temática detrás de la

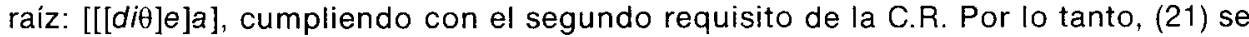
aplicará antes que (20), produciendo en (19A) la forma dika, que luego se hará diga por la aplicación de otras reglas. La regla (20) no podrá aplicarse y generar una forma incorrecta como *[[[dik]a]a]. La predicción de la C.R. es, pues, suficiente para generar formas irregulares.

Se puede demostrar que la inserción de la $1 .{ }^{a}$ persona de singular del Presente de Indicativo en el ciclo siguiente al de la vocal temática sigue los mismos principios que el del Presente de Subjuntivo.

Si tenemos una regla general de formación de $1{ }^{a}$ persona de singular de Presente de Indicativo, ésta podría enunciarse como en (22):

$$
\varnothing \rightarrow 0, /[V-]+V, \text { t.a p.s.P.I. }
$$

Sin embargo, por la C.R. y la relación disyuntiva en la que pueden entrar (22) y (18 aii), la regla (18aii), más específica, nos bloquea la aplicación de (22) con lo que perderíamos derivar la 1. ${ }^{a}$ p.s.P.I. para los verbos especiales, obteniendo la forma incorrecta *dik.

Por lo tanto, no es suficiente tener sólo una regla general de formación de $1 .^{\text {a }}$ p.s.P.l., sino que es necesario introducir una regla especial para ciertos verbos que son de derivación cero en el ciclo anterior. Esta regla morfológica puede enunciarse como en (23).

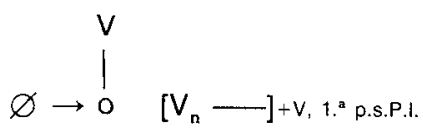

Donde $\mathrm{V}_{\mathrm{n}}=$ hacer, decir

La regla específica (23) entra en relación disyuntiva con la regla (22), más general. La regla (23) define un subconjunto de los contextos de (22), ya que la descripción estructural de (23) incluye propiamente a la de (22). La primera condición de la C.R. se cumple. El cambio estructural de (23) es distinto al de (22) ya que (23)

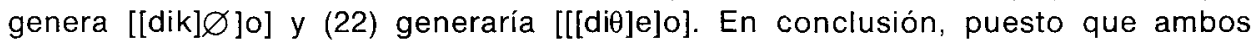
requisitos de la C.R. se cumplen, (23) se aplicará y bloqueará la aplicación de (22), evitando la forma incorrecta *[[[dik]o]o].

Hemos demostrado, pues, que la C.R. genera apropiadamente los casos particulares del Presente de Subjuntivo y del Presente de Indicativo en su $1 .^{a}$ persona de singular: dika $\rightarrow$ diga; diko $\rightarrow$ digo respectivamente.

Nótese que no podemos tener la formación del Presente de Subjuntivo y/o la de la $1 .{ }^{a}$ p.s.P.I. de los verbos especiales hacer y decir y de los regulares en el mismo nivel. La razón es que nada impediría la aplicación de la regla general (20) o (22) a las formas regulares salidas de la formación de vocal temática como, por ejemplo, 


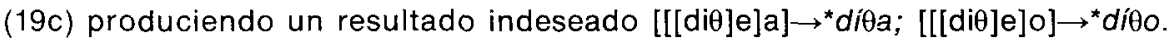

Es, por lo tanto, necesario que la regla de formación de Presente de Subjuntivo y la de $1 .{ }^{a}$ p.s.P.l. de los verbos especiales se produzca en un nivel distinto al de los mismos Tiempos y personas de los verbos no especiales. Si las reglas generales de formación de P.S. y $1 .{ }^{\mathrm{a}}$ p.s.P.I., (20) y (22) respectivamente, aplican en el nivel II pero no en el I y las reglas específicas sinónimas (21) y (23) respectivamente aplican en el nivel I pero no en el nivel II, entonces la C.R. dentro de esta teoría léxica nos da el resultado deseado. Veamos por qué.

Recuérdese que, por las condiciones (13i) y (13ii), todas las entradas provenientes del nivel I son entradas léxicas en el nivel Il y que estas entradas tienen una regla de identidad. Así, por ejemplo, puesto que existe una regla de identidad en el nivel II como la de (24), la regla específica de identidad del Presente de Subjuntivo entra en una relación disyuntiva con la regla sinónima del Presente de Subjuntivo de carácter general (20).

$$
\underset{+V \text {.P.S. }}{\left[V_{n}\right]} \underset{+ \text { V.P.S. }}{\rightarrow}\left[\mathrm{V}_{n}\right]
$$

Donde $\mathrm{V}_{\mathrm{n}}=$ hacer, decir.

Puesto que la descripción estructural de (24) incluye propiamente a la de (20), esto es, (24) define un subconjunto de los contextos de (20) y puesto que el cambio

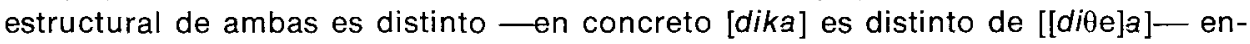
tonces (24) se aplicará antes bloqueando la aplicación de (20). Por lo tanto, bloqueará la formación del Presente de Subjuntivo de los verbos irregulares evitando

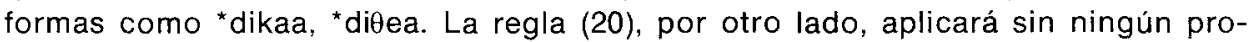
blema en todos los otros casos de los verbos regulares.

La misma demostración serviría para el caso de la $1 .{ }^{a}$ p.s.P.I.

Hemos demostrado que la teoria léxica junto con la C.R. genera adecuadamente tanto los casos particulares como dígo, díga, como los casos generales como dices, cueces, etc., sin necesidad de utilizar una inversión de orden en la aplicación de dos reglas dadas. Las formas especificas se forman a un nivel anterior que las formas generales, pero comparten los mismos tipos de procesos que las generales.

Veamos cuál es la interrelación de las dos reglas de las que hemos tratado inicialmente: Truncamiento y Desvelarización, en este léxico.

\section{Consecuencias teóricas}

La aplicación de las reglas generales de Presente de Subjuntivo y de 1. ${ }^{\text {ap.s.P.I. }}$ en el nivel II crea el contexto para la aplicación de la regla de Truncamiento, puesto que aquellas originan la coalescencia de dos vocales. La vocal temática que aparece en el nivel I ha originado la posible aplicación de la regla de Desvelarización en ese mismo nivel. Ahora, la aparición de la vocal del Subjuntivo Presente y de la 1. ${ }^{a}$ p.s. del P.I. originan el contexto para la aplicación en el nivel II de la regla de Truncamiento. Puesto que no hay razones para pensar que la regla de truncamiento tenga 
que aplicar en el nivel I y no hay razones para que no aplique en el nivel II, podemos concluir que mientras la regla de Desvelarización tiene que aplicar en el nivel l, la regla de Truncamiento puede aplicar máximamente en el nivel $\mid{ }^{13}$.

Parece, pues, que la ordenación Desvelarización precediendo Truncamiento viene motivada por razones independientes y no por un orden extrínseco ad hoc asociado a entradas léxicas concretas.

Nótese que una de las consecuencias de este análisis es que en un verbo como marcar o sacar en su forma de Presente de Subjuntivo se puede ahora explicar por qué no tiene la forma *sa日emos. Su derivación aparece en (25).

$$
\text { [[[[sak] a] e] mos] }
$$

Nivel I

$$
\text { Nivel II }
$$

$$
\text { Desv. - }
$$

Trunc. $\varnothing$

O.R.

$$
\text { [sakémos] }
$$

Si Desvelarización fuera una regla del nivel II tendríamos que ordenar extrínsecamente Desvelarización antes que Truncamiento. Si hacemos esto, entonces tenemos una forma de bloquear la aplicación de Desvelarización, pues la regla no encontraría su descripción estructural apropiada. Si no la ordenáramos extrínsecamente dejando que se aplique después de Truncamiento, la Condición de Ciclo Estricto no podría bloquearla, puesto que esta regla cumple la condición de aplicarse en el mismo ciclo en el que se ha introducido información relevante. A saber, la aplicación de Truncamiento ha dejado en contacto la velar sorda con la vocal anterior permitiendo así la aplicación de Desvelarización. Veamos esto en la derivación (26).

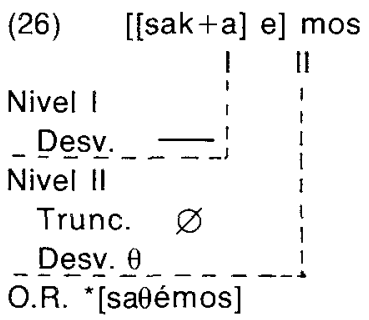

13 La Condición de Ciclo Estricto con una formulación un tanto simplificada pero suficiente para nuestra discusión es enunciada por Kiparsky (1982, p. 154) de la siguiente forma:

Strict Cycle Condition (scc)

a. Cyclic Rules apply only to derived representations.

b. Def.: A representation $\varnothing$ is derived w.r.t. rule $R$ in cycle $j$ iff $\varnothing$ meets the structural analysis of R by virtue of a combination of morphemes introduced in cycle $\mathrm{j}$ or the application of a phonological rule in cycle $\mathrm{j}$. 
Sin embargo, la Condición de Ciclo Estricto tendría que ser invocada si en el ciclo siguiente se volviera a aplicar el mismo orden: Desvelarización antes que Truncamiento,

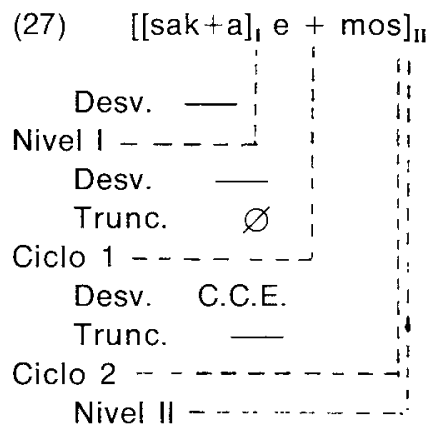

O.R.

[sakémos]

Nuestro análisis, basándose en la necesidad independiente de no permitir la regla de Desvelarización en el Nivel II y de necesitarla en el Nivel I, junto con la necesidad de tener la vocal de Tiempo y Modo en el Nivel II pero no en el I, nos permite prescindir de la CEE, y de tener que dar un orden extrínseco dentro de un mismo nivel en el que, en principio, se podría permitir el inverso.

Pasaremos ahora a una cuestión técnica en la formulación de la regla de Desvelarización.

Si suponemos que los corchetes sustituyen a los antiguos lindes de morfema, dado el principio (13i), la regla de Truncamiento, tal y como está formulada en (1) (Harris 1969, 1973), no podría aplicar en el Nivel II, puesto que la linde morfemática entre la raíz y la vocal temática serían borradas en el Nivel 1. Por lo tanto, (1) no podría aplicar en (28a) y aplicaría mal en (28b):

(28a)

$$
\begin{aligned}
& \text { [[[saka }] \text { e }]_{\sharp 1} \text { mos] } \\
& \text { Nivel II }
\end{aligned}
$$

Trunc.

O.R. ...

*[sakaémos] (28b) $\left[\left[\left[\text { saka }_{1}\right] \text { e }\right]_{\text {II }}\right.$ is $]$

Trunc. $\varnothing$

O.R

*[sakáys]

Hay, pues, razones para que dentro de este marco de trabajo la regla de Truncamiento sea reformulada como en (29).

$$
\begin{aligned}
& \text { Truncamiento Revisada. } \\
& \mathrm{V} \rightarrow \varnothing /-\mathrm{J}]
\end{aligned}
$$

La regla (29) se aplica correctamente ahora a (28a) y (28b). Incluso en un caso como el de (30) el resultado es el correcto. Obsérvese que la formulación de (1) presenta una ambigüedad en el caso de encontrar tres vocales en secuencia. La regla tendría que tener una restricción específica que asegurara que su aplicación se efectúa de 
izquierda a derecha en la palabra pero no de derecha a izquierda. Este problema desaparece en nuestro análisis, como se demuestra en (30a)

(30a) $\left[\left[[\text { marea }]_{1} e\right]_{\text {II }}\right.$ is]

Nivel II

Trunc. R.

$\varnothing$

Acento

Penúlt.

O.R.

[mareéys] (30b) $[$ mare $+a+e+i s]$

Trunc. $\left\{\varnothing^{*} \varnothing\right\} \begin{aligned} & \text { Iz. Der. } \\ & \text { Der. Iz. }\end{aligned}$ $\begin{array}{l}\text { Acento } \\ \text { Penúlt. }\end{array}$ *á $\left.^{\text {é }}\right\}$

$\stackrel{\text { O.R. }}{\left.\left[\begin{array}{c}\text { mareéys } \\ { }^{*} \text { mareáys }\end{array}\right\}\right]}$

Con la formulación de (29) y con la formación endocéntrica de las formas verbales evitamos el tener que añadir condiciones a las reglas de la fonología.

\section{Discusión}

Antes de pasar a la conclusión, sería conveniente tratar dos cuestiones teóricas de carácter global. Una está relacionada con la evaluación de las alternativas que, hasta ahora, se han propuesto para dar cuenta de las irregularidades que aquí nos ocupan. La otra aborda el problema de si la inflexión debe hacerse en el léxico o en la sintaxis.

\subsection{Estipulaciones únicas}

A nadie se le escapa el que introducir en la gramática un nuevo y desconocido proceso, el de selección de ordenaciones, tan sólo para estos verbos en toda la gramática del español parece enormemente costoso ya que la teoría se debilita de una forma incalculable. La pregunta que deberíamos hacernos en esta hipótesis es ¿por qué no hay más casos de ordenación doble?, y si los hubiera ¿cuáles serían sus implicaciones? Por el momento, hasta que no se demuestre que las ordenaciones dobles son un proceso «natural» y necesario en otras muchas lenguas, la hipótesis de su necesidad en español es altamente cuestionable.

Esta hipótesis, llamémosla aquí «la hipótesis transformacionalista», debe evaluarse con la "hipótesis lexicalista" que es la que defendemos en nuestro trabajo. En la primera, nos vemos obligados, en aras de mantener una uniformidad en la estructura profunda, a complicar el componente transformacional de una forma altamente costosa. En la segunda, las diferencias superficiales se derivan no de dos modos distintos de ordenación transformacional sino de dos estructuras iniciales distintas que son independientemente necesarias en el léxico (cfr. [freído]/[frí $\varnothing$ to]; [dik $\varnothing \theta$ yón]/[de日ir]; [abyér $\varnothing$ to]/[abrír] etc.). 
Nótese que la no aparición de $[\theta]$ en cualquiera de estos contextos no viene como consecuencia de que haya Truncamiento, como debería predecir la «hipótesis transformacionalista", ya que la forma [dik $\theta$ yón] no tiene $[\theta]$ en la raíz y sin embargo esta peculiaridad no puede derivarse de la aplicación de Truncamiento pues no ha habido contexto para que el proceso se aplicara. Por el contrario, la hipótesis lexicalista predice que la no aparición de [ $\theta]$ viene como consecuencia de la existencia de derivaciones temáticas vacías segmentalmente en la morfología léxica que pueden bloquear otros procesos. Así, tanto la regla de Desvelarización en [dik $\varnothing$ $\theta y o ́ n][d i ́ g \varnothing a]$ etc., como la regla de Sonorización en [fríøto], [mwér $\varnothing$ to] etc., se ven bloqueadas no por su interrelación con Truncamiento, sino por la presencia de una estructura temática vacía.

Dicho de otro modo, si pudiéramos deducir que siempre que no aparece [ $\theta$ ] o [d] es porque Truncamiento ha aplicado antes de los otros dos procesos, entonces tendríamos una razón válida para pensar que Truncamiento puede ser un proceso bloqueador de otros: Desvelarización y Sonorización. Lo único que podemos deducir es que siempre que no aparece la vocal temática con su representación segmental ciertos procesos, que necesitan esa vocal segmental, no pueden aplicarse.

En conclusión, una estipulación única en la hipótesis transformacionalista (la ordenación doble) se contrapone a una estipulación única en la hipótesis lexicalista (dobles estructuras temáticas). Sólo la estipulación lexicalista parece poder abarcar más hechos de una forma más uniforme.

\subsection{Arbitrariedad contextual}

Ahora bien, nos falta una explicación de porqué ciertas formas verbales en la hipótesis lexicalista, tal y como el Presente de Indicativo en su primera persona o el Presente de Subjuntivo, son las que producen estas vocales temáticas vacías y no por ejemplo en imperfecto de Indicativo. Una cosa parece clara y es que si las irregularidades verbales se distribuyen en (la $1 .^{a}$ persona de sing. del) Presente de Indicativo de la [-I Conj.], éstas también se producen en el Presente de Subjuntivo: (Cf. veo/vea, salgo/salga, conozco/conozca, traigo/traiga).

Esta agrupación temporal no es sin embargo un hecho aislado en la morfología verbal del español. Así, si el Pretérito es irregular en la raíz, las mismas irregularidades aparecen en las raíces de los Imperfectos de Subjuntivo y el Futuro de Subjuntivo: cf. dije, dijera, dijese, dijere; supe, supiera, supiese, supiere; puse, pusiera, pusiese, pusiere, etc.

No cabe duda de que aún nos falta mucho por entender: por qué ciertos tiempos se unen en las irregularidaes y otros no. Por lo tanto, la arbitrariedad de los contextos en los que aparece la vocal temática vacía no es un hecho tan arbitrario en la morfología española, sino que parece pertenece a un problema más general que hay que investigar aún.

\subsection{Arbitrariedad de «n»}

Otro problema que aparece en la hipótesis lexicalista es que las reglas (18a) y 
(21) requieren un diacrítico arbitrario " $n$ ". Parece claro que « $n$ » representa un subconjunto de verbos que de alguna forma se comporta de forma distinta a otro subconjunto. De la misma forma, ciertos procesos como el de Diptongación de las raices verbales aparece en un subconjunto de verbos $V_{n}$ : (sentir/siento) pero no en otro $V$ : (servir/ ${ }^{*}$ Siervo, sirvo), o en un subconjunto de tiempos $T_{n}$ : (partiera/partiendo) pero no en otro $T$ : (partir/partido) o en un subconjunto de personas $P_{n}$ : (partieron) pero no en otro P: (*partiemos).

Por lo tanto, nos encontramos con el hecho, por ahora arbitrario, de que ciertos procesos afectan a subconjuntos de categorías morfológicas así como a un subconjunto de raíces. Nada en la teoría que utilizamos puede «explicar» esta selección aún.

\subsection{Duplicidad de reglas}

En la mente de todos está el introducir las arbitrariedades como formas no derivadas. Así, en una hipótesis lexicalista más fuerte que la que he tratado de desarrollar aquí, podríamos tener como formas léxicas no derivadas [digo] 1."P. Sing. P.l. [ago] 1. a. s. sing. P.1. y [diga] P.S. [aga] P.S.

Puesto que tenemos la Condición de Resto (14) y las reglas de identidad (12ii), en el Nivel I tendriamos, en vez de (18ai), la regla de identidad (31) aplicándose en disyunción con (18b). En el Nivel II tendríamos, en vez de (21), la regla (31) ordenada en disyunción con (20)

$(24)=(31) \quad\left[V_{n}\right]_{+}$v. P.s. $\rightarrow\left[V_{n}\right]_{+v}$. P.s.

Donde $V_{n}=/$ diga/, /aga/.

Con esto, no hemos reducido el número de aplicaciones de reglas, puesto que independientemente en cada nivel una regla como (31) tiene que crearse para asegurar su precedencia sobre las otras formaciones verbales del nivel en cuestión. En cualquier caso la duplicidad de reglas $(31)$ y $\left(18^{b}\right)$ o de $(31)$ y $(20)$ en esta teoría lexicalista (fuerte o débil) no puede evitarse.

Por lo tanto, con la teoría lexicalista fuerte hemos aumentado el número de formas léxicas no derivadas sin haber reducido el número de reglas del léxico. Claramente bajo un criterio de simplicidad la hipótesis lexicalista débil parece preferible ${ }^{14}$.

En cuanto a la segunda cuestión, tan sólo muy brevemente, apuntar que nada parece ir en contra de que la inflexión se haga en el léxico: (véase Jensen \& Jensen (1984)). Las peculiaridades de concordancia de modos, tiempos, personas y géneros deben tratarse antes de que se efectúe la inserción.

14 Agradezco a Jim Harris el haberme hecho notar este síndrome de síntomas (461, 2, 3, 4) que parecen llevar a una descripción costosa y que aquí hemos discutido brevemente. Cualquier error es mio. 


\section{Conclusión}

A lo largo de este trabajo hemos demostrado que no parece haber necesidad de evocar una ordenación inversa a la "normal" para explicar la ausencia de desvelarización en formas como digo, diga, etc. Hemos ofrecido una alternativa dentro de un marco más restringido, pudiendo con ello dar cuenta de alternancias, no sólo en formas verbales, sino también en formas nominales con un tratamiento uniforme morfológico y fonológico. Hemos librado a la gramática de tener que imponer en estos casos órdenes extrínsecos o de tener que especificar el orden para formas léxicas determinadas. Nuestro análisis trata de que la ordenación de las reglas en cuestión venga derivada de hechos intrínsecos a la gramática más que de factores de «normalidad», "preferibilidad" u otras consideraciones que son, claramente, de orden no estrictamente interno a la gramática. Con este análisis creemos estar encaminando el estudio de la morfología y fonología del español de forma tal que contribuya a restringir el conjunto de gramáticas posibles.

\section{Referencias bibliográficas}

Allen, Margaret Reece (1978), Morphological Investigations. Tesis Doctoral. University of Connecticut.

Aronoff, Mark (1976), Word Formation in Generative Grammar. Linguistic Inquiry Monograph, 1. Cambridge Mass, M.I.T. Press.

Bach, E. y R. Harms (eds.) (1968), Universals in Linguistic Theory. New York, Holt. Brame, M. K. (1972), The Segmental Cycle. En Brame (ed.).

- (Ed.) (1972), Contributions to Generative Phonology. Austin University of Texas Press.

- (1974), The cycle in Phonology: stress in Palestinian, Maltese and Spanish. Linguistic Inquiry 5: 39-60.

Chomsky, Noam; M. Halle and F. Lukoff (1956), On accent and juncture in English. En For Roman Jakobson.

Chomsky, Noam and Morris Halle (1968), The Sound Pattern of English. New York; Harper and Row. Versión española de la parte I y IV en Principios de Fonologia Generativa. Editorial Fundamentos, 1979.

Fujimura, O. (ed.) (1973), Three Dimensions in Linguistic Theory. Tokyo TEC.

Goldsmith, John (1976), Autosegmental Phonology. MIT Tesis Doctoral. Reproducida por Indiana University. Linguistics Club.

Halle, Morris (1973), Prolegomena to a Theory of Word Formation. Linguistic Inquiry 4: 3-16.

- (1978), Formal vs Functional Considerations in Phonology. Indiana University Linguistics Club.

Harris James, W. (1969), Spanish Phonology. Cambridge Mass. The MIT Press. Versión Española (1975) Fonología Generativa del Español. Planeta. Barcelona.

- (1973), On the ordering of certain Phonological rules in Spanish. En Kiparsky \& Anderson (Eds.). Versión española (1975) Fonologia Generativa del Español. Apéndice $D$. 
Jackendoff, Ray (1975), Morphological and Semantic Regularities in the Lexicon. Language 51: 639-671.

Jensen, J. y Strong-Jensen, M. (1984), “Morphlogy is in the lexicon!», L./ 415, 3, pp. 474-97.

Kean, M.L. (1974), The Strict Cycle in Phonology. Linguistic Inquiry 5: 179-203.

Kielhorn, F. (1880), The Vyākarana-Mahābhasya of Patañjali I-III. Poona.

Kiparsky, Paul (1968), Linguistics Universals and Linguistic Change. En Bach \& Harms (Eds.), 171-202.

- (1968-1973), How abstract is Phonology? Indiana University Linguistics Club. También en Fujimura (Ed.).

- (1973), Abstractness, opacity, and global rules. En Fujimura (Ed.) 57-86.

- (1973a), Elsewhere in Phonology. En Kiparsky \& Anderson (Eds.). También en Indiana University Linguistics Club.

- (1979), Metrical Structure Assignment is Cyclic. Linguistic Inquiry 10: 421442.

- (1982a), From Cyclic Phonology to Lexical Phonology. En Van der Hulst \& Norval Smith (Eds.) 131-175.

- (1982b), Lexical Morphology and Phonology. En Is Yang (Ed.) Linguistics in the Morning Calm. Hanshin Seoul.

- and Anderson, S. (Eds.) (1973), Festschrift for Morris Halle. New York. Holt Reinhart and Wiston.

Liberman, M. y A. Prince (1977), On stress and Linguistic Rhythm. Linguistic Inquiry 8: $249-336$.

Mascaró, Joan (1976), Catalan Phonology and the Phonological Cycle. Tesis Doctoral MIT. Reproducida por Indiana University Linguistics Club (1978).

McCarthy, John (1979), On stress and syllabification. Linguistic Inquiry 10: 443-465.

- (1981), A Prosodic Theory of Nonconcatenative Morphology. Linguistic Inquiry 12: 373-418.

Mohanan, K. P. \& Tara Mohanan (1984), Lexical Phonology of the consonant System in Malayalam. L.I. 15: 575-602.

Selkirk, E. O. (1982), The syntax of words. Linguistic Inquiry Monograph 7 Cambridge Mass., MIT Press.

Siegel, Dorothy (1974), Topics in English Morphology. Tesis Doctoral MIT.

Van der Hulst and Norval Smith (Eds.) (1982), The structure of Phonologycal Representations (Part I) Foris. 